\title{
Optical and Structural Characterization of a-Si Thin Film Solar Absorbers
}

\author{
George Atanasoff*, Amogh Mudgal*, Li-Chung Lai** and Wen-An Chiou** \\ * AccuStrata, Inc., University of Maryland TAP, 387Technology Dr., College Park, MD 20742 \\ ** NISP Lab, NanoCenter, University of Maryland, College Park, MD 20742-2831
}

With the increase demand of energy and the limited resources on the Earth, research of solar cell, a device that converts the energy of sunlight directly into electricity by the photovoltaic effect, has been accelerated in the last decade. Real time information about the film optical constants and material bandgap and their change during the deposition process is very important for most of the solar panel manufacturers since it can be directly correlated to the final solar panel efficiency. We have developed a real time optical control system for thin film deposition. The system performs derivation of the film thickness and its uniformity, the deposition rate, optical constants - n\&k and the material bandgap as the film is deposited on a stationary panel or on a moving flexible roll. While demonstrating the advantage of real time optical monitoring, some film parameter nonhomogeneity was found. This research was thus undertaken to investigate the possible causes of film inhomogeneity using TEM.

A variety of amorphous silicon (a-Si) solar absorbers during their deposition was monitored. The films were deposited by PECVD in a superstrate process on glass panels with already pre-deposited SnO layer (NSG - Pilkington) as transparent conductive oxide (TCO) layer. The optical monitoring was performed by monitoring the specular reflectance in a broad spectral region and, in parallel, at one fixed wavelength. The broadband spectral region was chosen to be $400 \mathrm{~nm}$ to $1000 \mathrm{~nm}$. The fixed wavelength measurement was performed at $\lambda_{0}=850 \mathrm{~nm}$. The film optical constant determination method was based on the evaluation of the envelope curves connecting the reflectance minima and maxima at one fixed wavelength $[1,2]$. The optical absorption coefficient $\alpha$ was then calculated, which was used to calculate the film material band gap by using some known linear extrapolation such as Cody's extrapolation [3]. The TEM sample was prepared using conventional technique by sandwiching two film/substrate layers following by grinding, polishing and brief ion milling for 30 minutes to minimize possible artifact from ion beam thinning.

Fig. 1 shows the refractive index $n$ and the extinction coefficient $k$ of the growing film as a function of film thickness. During the first $60-70 \mathrm{~nm}$ the film grows as with index of refraction was about 4.07 at wavelength $\lambda_{0}=850 \mathrm{~nm}$ and non-detectable absorption coefficient which is typical for a-Si thin film. As the film thickness increases the film refractive index decreases by about $10-12 \%$ and its absorption coefficient increases to about $3000 \mathrm{~cm}^{-1}(k \sim 0.02)$. This optical constant inhomogeneity was not typical for an amorphous Si film and, thus, TEM investigation of the samples was undertaken. Fig. 2 shows that the film material band gap decreased during the deposition by about $0.05 \mathrm{eV}$. These measurements showed inhomogeneity in optical constants and material band gap in depth of the amorphous (a)-Si films. Fig. 3 reveals a cross-section TEM view of the amorphous-like Si film with a typical broad diffraction ring. The broad electron diffraction ring suggests the amorphous and/or nano-crystalline nature of the film. HRTEM images (Fig. 4), however, depict numerous "short-range" order lattice fringes (circles) scattered in the a-Si thin film. Those poorly ordered nano-crystalline phases, though the mechanism of formation is not fully understood, in the amorphous Si film could cause the reduction of the refractive index and material band gap as well as the increase of the extinction coefficient. The refractive index of the crystalline phase is lower than that of the amorphous material and as a result the effective refractive index of the material is reduced. It is also well known fact that the band gap of the polycrystalline silicon is 
lower than that of the amorphous material. TEM study suggested that the formation inhomogeneous nano-crystalline phase in the amorphous film effectively reduces the film material band gap.

[1] L. Landau and E. Lifchitz, Quantum Mechanics, Non-Relativistic Theory, Course in Theor. Physics, Vol. 3, Pergamon, London (1958)

[2] D. Poitras and Ludvic Martinu, Appl. Optics, 37, 19, 4160 - 4167 (1998)

[3] G.D. Cody et al., Sol. Energy Mater., 8, 231-238 (1982)

[4] The authors are thankful to Oscar von Bredow, Rodd Pribik and Hasso von Bredow for their help. This work was supported by NSF SBIR, DoE Supply Chain Program, and NISP Lab (MRSEC, NSF supported Shared Facility), NanoCenter at the University of Maryland.

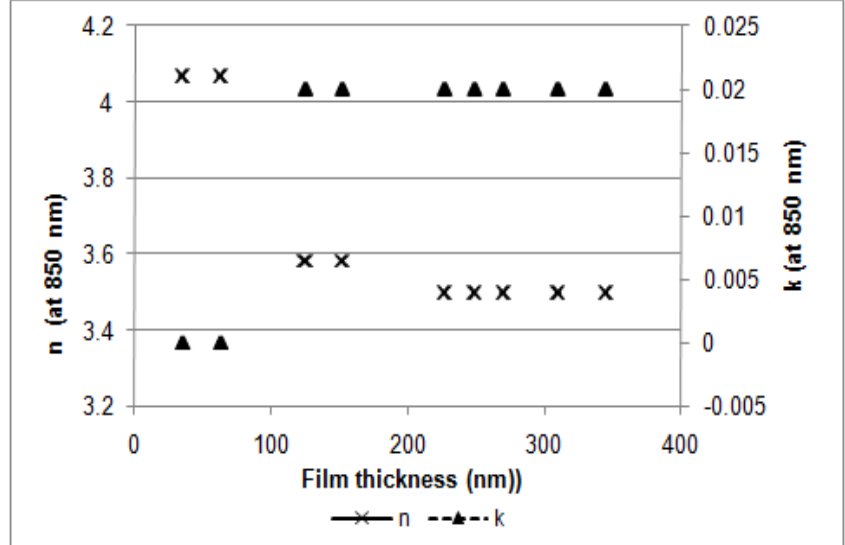

Fig. 1. Typical refractive index $n$ and the extinction coefficient $k$ of the growing film as a function of film thickness

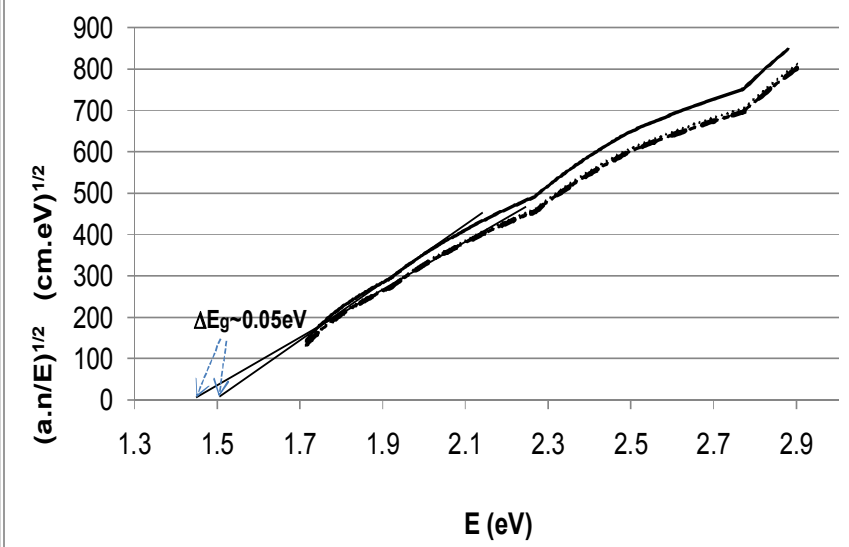

Fig. 2. Change in the film band gap during deposition.

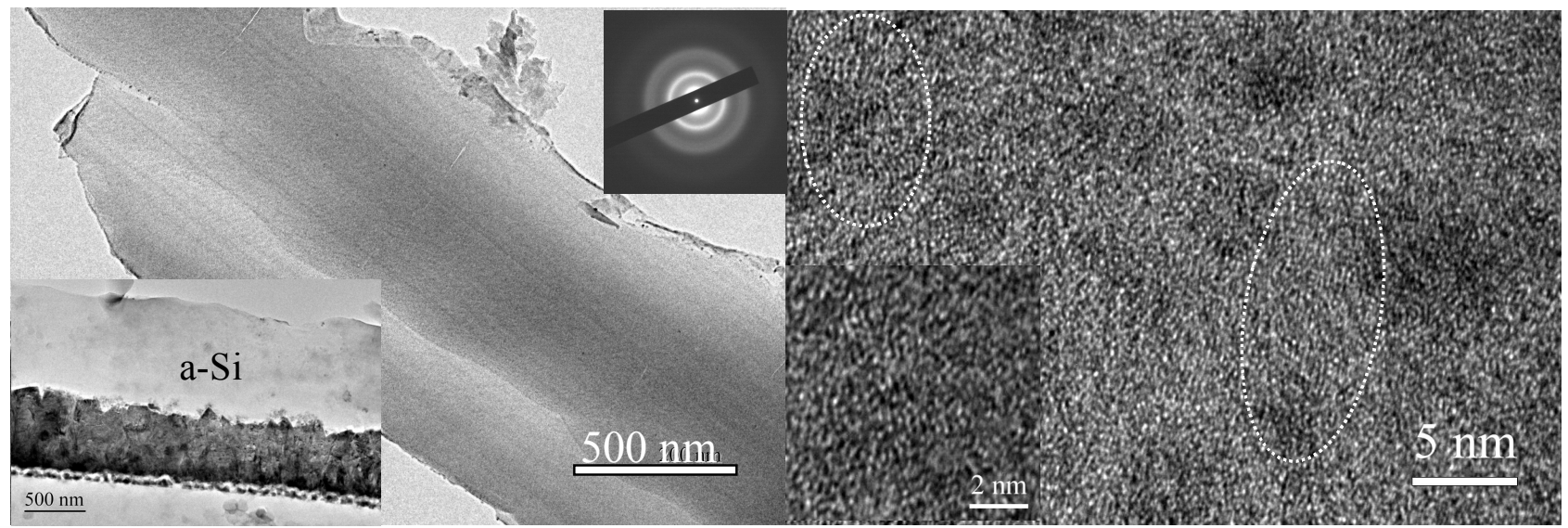

Fig. 3. Cross-sectional TEM micrograph of the a-Si thin film showing some parallel dark lines that probably resulted from pulse deposition. Low magnification crosssectional TEM view reveals the a-Si film coated on Sn oxide layer which overlaid on a silicate glass.
Fig. 4. High resolution TEM micrograph of aSi film showing numerous very short-range order lattice fringes (circle areas) from place to place. These poorly ordered nanocrystallites formed during the film deposition could cause the reduction of the refractive index and material band gap as well as the increase of the extinction coefficient. 